\title{
Musivaria HD: proyecto para el estudio, investigación y difusión de los mosaicos romanos tardoantiguos de la Península Ibérica desde las Humanidades Digitales
}

Musivaria HD: project for the study, research and dissemination of the late-ancient Roman mosaics of the Iberian Peninsula from the Digital Humanities

\author{
Beatriz Garrido-Ramos ${ }^{\mathrm{a}}$ \\ a Facultad de Geografía e Historia, UNED, bgarridoramos@gmail.com
}

\begin{abstract}
Resumen
Los mosaicos tardoantiguos conforman un importantísimo patrimonio material que contribuye sin duda al conocimiento de la cultura de época romana. Musivaria HD es un proyecto destinado al estudio, la investigación y la difusión de los mosaicos de Hispania desde la perspectiva de las denominadas "Humanidades Digitales". Recopila un buen número de reconstrucciones $3 D$, vídeos y material gráfico. Se trabajan los metadatos para una mejor descripción de las obras y se aplica la clasificación Iconclass para la analizar la iconografia de los mosaicos. La web es única en su campo por albergar un conjunto de mosaicos que habitualmente no se encuentran concentrados ya que suele recurrirse a su estudio de forma aislada por zonas. Dispone de un menú principal con sus correspondientes apartados y subapartados, de manera que facilita la navegación y la experiencia de usuario, y le presenta de forma intuitiva y rápida toda la información existente en la página. Musivaria HD combina además las tecnologías PHP, HTML5, CSS3 y JavaScript con el Diseño Web Adaptativo (Responsive Web Design) destinado a su visualización en cualquier dispositivo.
\end{abstract}

Palabras clave: Musivaria romana tardoantigua; Humanidades Digitales; Hispania; Iconclass; iconografia; metadatos; proyecto; difusión; patrimonio cultural;

\footnotetext{
Abstract

The late-ancient mosaics make up a very important material heritage that undoubtedly contributes to the knowledge of the culture of Roman times. Musivaria HD is a project aimed at the study, research and dissemination of Hispania mosaics from the perspective of the so-called "Digital Humanities".
} 
Musivaria HD: proyecto para el estudio, investigación y difusión de los mosaicos romanos tardoantiguos de la Península Ibérica desde las Humanidades Digitales

It compiles a good number of $3 D$ reconstructions, videos and graphic material. The metadata is worked on for a better description of the works and the Iconclass classification is applied to analyze the iconography of the mosaics. The web is unique in its field because it houses a set of mosaics that are not usually concentrated, since they are usually studied in isolation by zones. It has a main menu with its corresponding sections and subsections, in such a way that it facilitates navigation and the user experience, and presents all the information on the page intuitively and quickly. Musivaria HD also combines PHP, HTML5, CSS3 and JavaScript technologies with Responsive Web Design for viewing on any device.

Keywords: Late ancient Roman musivaria project in Hispania, Digital Humanities, Iconclass, metadata, dissemination of cultural heritage 


\section{Introducción}

Esta comunicación abordará brevemente la web Musivaria $H D$ como resultado de la investigación de la tesis doctoral llevada a cabo por la autora. Se hará un recorrido por las características, el contenido y las novedades que aporta al ámbito de la musivaria romana tardoantigua en Hispania. Todo ello, como se ha expuesto previamente y sin querer profundizar en deficiones ni marcos teóricos puesto que no es el cometido de este texto, desde la perspectiva multidisciplinar y emergente de las Humanidades Digitales (en adelante HD).

\section{Proyecto Musivaria HD: una nueva aproximación metodológica desde las Humanidades Digitales}

La musivaria tardoantigua ha resultado una fuente de información importantísima para el estudio y la investigación de época romana, tanto en Hispania como en el resto del imperio.

Por ello, y con motivo de la investigación doctoral realizada previamente, se consideró imprescindible poner a disposición de la comunidad científica y del público en general todos los datos disponibles y recopilados durante el proceso. A ello se añade la necesidad de difundir la metodología novedosa aplicada así como los resultados obtenidos.

Los objetivos del proyecto son diversos pero, entre otras cuestiones, se centraron en los siguientes puntos:

- Contribuir al estudio e investigación de la musivaria existente en la Hispania romana desde otra perspectiva totalmente novedosa como es la de las HD que difiere por completo de la habitual.

- Estudiar los mosaicos de una forma interdisciplinar ${ }^{1}$ aunando campos tan dispares como la Geografía (SIG), la Historia del Arte (Iconografía), la Filología (estudio taxonómico, metadatos, etc.), las Matemáticas (Geometría), etc. mediante la utilización de herramientas de distinto tipo, estándares y sistemas de clasificación diversos.

- Detectar patrones concretos que a simple vista podrían pasar desapercibidos.

- Formular hipótesis y plantear nuevas preguntas de investigación.

- Difundir la información obtenida y recopilada durante la investigación relativa a los mosaicos tardoantiguos repertorizados.

\footnotetext{
${ }^{1}$ Para ampliar información véase: https://musivariahd.com/geografia-del-mosaico-y-triangulacion-de-datos.html
} 
Musivaria HD: proyecto para el estudio, investigación y difusión de los mosaicos romanos tardoantiguos de la Península Ibérica desde las Humanidades Digitales

El sistema fue diseñado siguiendo la Norma CIDOC-CRM². El Modelo de Referencia Conceptual $^{3}$ (CRM) se trata de una ontología formal aplicada en el ámbito del Patrimonio Cultural. Es llevada a cabo por un grupo de trabajo del Comité de Documentación que se desarrolla en el Consejo Internacional de Museos (ICOM) y, en consecuencia, es el modelo que se ha tenido presente a la hora de desarrollar la web y el contenido que alberga. Todo lo anterior conducirá a la puesta en valor del patrimonio material y cultural relativo a los mosaicos romanos existentes en la Península Ibérica que sin lugar a dudas merecen toda nuestra atención.

\subsection{Visualización de datos}

Con el acceso a una mayor cantidad de información "surgen nuevos problemas para su tratamiento, siendo necesarios nuevos métodos y tecnologías. La informática se encarga de definir estos procedimientos y tecnologías que nos facilitan el manejo de grandes volúmenes de información de una manera amigable" (Rincón, 2015: 2). A este respecto hay que exponer que la parte de visualización de los datos (representación gráfica de la información y de los datos) es de especial relevancia en cualquier investigación, independientemente del tipo o ámbito del que se trate. Lev Manovich alude a la visualización de datos indicando que "nos permite ver los patrones y estructuras que subyacen en conjuntos de datos aparentemente arbitrarios" (Manovich, 2008: 130). Por lo que respecta al análisis visual de datos, según Thomas y Cook (2005) los "Análisis Visuales" o "Visual Analythics" son "la ciencia del razonamiento analítico facilitada por interfaces visuales interactivas".

\subsubsection{Gráficos y estadísticas}

En la web se pueden encontrar distintos gráficos y estadísticas en relación a dos cuestiones importantes: en primer lugar, los que se refieren a los propios mosaicos repertorizados ${ }^{4}$; en

\footnotetext{
${ }^{2}$ Tal y como se expone en la web: "Se ha desarrollado de una manera que tiene la intención de promover una comprensión compartida de la información del patrimonio cultural al proporcionar un marco semántico común y extensible para la integración de la información del patrimonio cultural basada en la evidencia. Está destinado a ser un lenguaje común para que los expertos en el dominio y los implementadores formulen requisitos para los sistemas de información y sirva como guía para las buenas prácticas de modelado conceptual. De esta forma, puede proporcionar el "pegamento semántico" necesario para mediar entre diferentes fuentes de información sobre el patrimonio cultural, como la publicada por museos, bibliotecas y archivos" (Traducción de la autora). Véase: http://www.cidoc-crm.org/

${ }^{3}$ Tal y como se expone en la web el CRM es una "herramienta teórica y práctica para la integración de información en el campo del patrimonio cultural. Puede ayudar a los investigadores, administradores y el público a explorar preguntas complejas con respecto a nuestro pasado a través de conjuntos de datos diversos y dispersos. El CIDOC CRM logra esto al proporcionar definiciones y una estructura formal para describir los conceptos y relaciones implícitos y explícitos utilizados en la documentación del patrimonio cultural y de interés general para la consulta y exploración de dichos datos. Estos modelos también se conocen como ontologías formales. Estas descripciones formales permiten la integración de datos de múltiples fuentes de forma independiente del software y del esquema" (Traducción de la autora). Para ampliar información véase: http://www.cidoc-crm.org/

${ }^{4}$ Véase: https://musivariahd.com/graficos-de-musivaria-hd.html
} 
segundo lugar, los de los resultados obtenidos de TaxMOS $H D^{5}$ - Taxonomía para la investigación y difusión de la iconografía musivaria romana desde las Humanidades Digitales. Creada por la autora, se espera que la taxonomía resulte particularmente útil para el análisis de la información sobre iconografía y mitología romanas, las principales técnicas constructivas en Hispania y el tipo de decoración de sus mosaicos, así como las herramientas, métodos y estándares aplicados a la Historia del Arte desde el campo de las Humanidades Digitales.

\subsection{Búsqueda de información. Vocabularios controlados y datos enlazados.}

Un conjunto de datos (dataset), como su propio nombre indica, no debe tratarse de forma aislada, porque carecería de sentido, sobre todo cuando nos referimos como en este caso a una base de datos (en adelante BD) cuya finalidad es cruzar los datos (como cualquier otra) sobre los mosaicos que la conforman para obtener determinados resultados en relación a un criterio de búsqueda concreto que permita establecer relaciones entre los distintos mosaicos. Este punto es especialmente importante porque con frecuencia se estudian de forma aislada, aspecto que dificulta la investigación y la extracción de conclusiones del conjunto. Analizar por tanto un conjunto de datos nos conduce a reflexionar y detectar posibles patrones en los mismos.

La web cuenta con un buscador ${ }^{7}$ interno por criterios de búsqueda sencilla, mediante la consulta de términos de cualquier campo de manera que recupere información existente en toda la web y la BD de Musivaria HD, o búsqueda avanzada, en la que el/la usuario/a podrá efectuar búsquedas en relación al municipio (de España o Portugal), tipo de lugar (yacimiento, villae, domus, indeterminado y otros casos de estudio), nombre de mosaico, tipo de mosaico (geométrico o figurativo) y descriptores o palabras clave ${ }^{8}$ (metadatos por los que se clasificaron previamente los mosaicos repertorizados).

Finalmente, hay que exponer, como indica Lina Nagel, que "la aplicación de estándares internacionales en el registro de colecciones es fundamental” (VV.AA., 2008: 49). Nagel prosigue y se refiere a la tecnología indicando que "cada vez más la tecnología nos exige, para una búsqueda y recuperación de información más eficiente, normalizar la terminología usada en el registro, descripción y clasificación de objetos culturales" (VV.AA., 2008: 49).

\footnotetext{
5 Véase: https://musivariahd.com/graficos-de-taxmos-hd.html

${ }^{6}$ Esta taxonomía sobre la investigación y difusión de la iconografia musivaria en la Hispania romana desde la perspectiva de las Humanidades Digitales ha sido desarrollada para intentar subsanar la falta de unificación de la información relativa a esa rama de la Historia del Arte que se encuentra con frecuencia dispersa y sin estandarizar, con el fin de que pueda ser utilizada por historiadores e investigadores para llevar a cabo estudios sobre la misma, así como por la comunidad de humanistas digitales a la que puede resultar de interés por tratarse de un ejemplo de aplicación de las HD a la Historia del Arte. La taxonomía tiene como principal objetivo difundir la cultura material que conforma la musivaria romana tardoantigua de la Península Ibérica, así como estructurar y difundir a su vez la información referente a las Humanidades Digitales y a la iconografía musivaria, tema sobre el que versa la tesis doctoral de la autora. Véase: http://www.vocabularyserver.com/taxmos/index.php

${ }^{7}$ Véase: https://musivariahd.com/buscador.html

${ }^{8}$ Listado de 739 palabras clave o descriptores: https://musivariahd.com/listado-de-descriptores-o-palabras-clave.html
} 
El trabajo con vocabularios controlados permite una mejor recuperación de la información. En Musivaria HD se ha trabajado con el Diccionario Geográfico GeoNames ${ }^{9}$, el Tesauro AAT ${ }^{10}$ (Art \& Architecture Thesaurus) y el Sistema de Clasificación Iconclass, siendo este último en el que nos centraremos en el siguiente apartado.

\subsection{Iconclass: aplicación de un sistema de clasificación para el estudio y la investigación de la iconografía romana en Hispania}

Para llevar a cabo la búsqueda y recuperación de información de una forma más eficiente, se torna necesario normalizar la terminología usada en el registro, en la descripción y en la clasificación de objetos culturales.

Iconclass es un Tesauro, es decir, un recurso que trabaja un vocabulario controlado que facilita la clasificación del patrimonio cultural mediante la aplicación de un estándar internacional, que por otra parte no es algo nuevo, ya que desde hace tiempo se está aplicando en proyectos de humanidades (otro ejemplo de vocabulario es el Tesauro AAT del Getty Research Institute citado previamente).

Por lo que respecta al Sistema de Clasificación, en la web de Iconclass se expone que reúne 28.000 definiciones ordenadas jerárquicamente, cada una de las cuales consistirá en un código alfanumérico de clasificación (notación) y la descripción de la materia iconográfica (correlato textual).

En la web de Iconclass (accesible en inglés) puede encontrarse una explicación de la clasificación, así como sus principales características. En ella se expone que se trata de una clasificación Linked Open Data (LOD), en la que cada notación, se asocia con un Identificador Uniforme de Recursos (URI) que es único para ese encabezamiento (GarridoRamos, 2017b: 25).

Llegados a este punto, debemos responder a dos cuestiones importantes para comprender este punto y son las siguientes: ¿Por qué se ha utilizado Iconclass? ¿Por qué se ha escogido esta clasificación en lugar de otra? A la primera pregunta responderíamos que es un sistema de clasificación diseñado específicamente para Arte e Iconografía. A la hora de trabajar con imágenes es una de las opciones que puede barajarse es Iconclass, principalmente porque permite gestionar vocabularios controlados para la descripción o clasificación de iconos o figuras, tal y como se expone en la web del proyecto (Garrido-Ramos, 2019: 356).

\footnotetext{
9 BD con información geográfica que contiene nombres geográficos organizados en nueve categorías y 645 subcategorías. Los recursos de GeoNames se identifican por sus correspondientes URIs, los cuales permiten acceder a información disponible en una Wiki o bien a su descripción en RDF (Resource Description Framework), siguiendo la ontología de GeoNames expresada en OWL. A través de la URL de los artículos de Wikipedia enlazados a la descripción RDF, los datos GeoNames se reenlazan a los de DBpedia y a otras fuentes RDF. Véase: http://www.geonames.org/

${ }^{10}$ Es un tesauro del Getty. En nuestro caso fue seleccionado y aplicado al estudio porque posee términos genéricos para describir arte y arquitectura (artes decorativas, cultura material, materiales de archivo, arqueología y conservación). Ha sido traducido al español como Tesauro de Arte y Arquitectura (TAA). https://www.aatespanol.cl/
} 
A continuación, expondremos brevemente cómo se ha procedido a aplicar este sistema de clasificación jerárquico para la descripción de los mosaicos repertorizados en la BD de nuestra web. En primer lugar comenzaremos por destacar que dispone de 10 categorías principales ${ }^{11}$ de las que parten otras subcategorías ${ }^{12}$ permitiendo profundizar en el nivel de análisis y descripción del objeto (temas y escenas representadas en los mosaicos en este caso) tanto como resulte necesario.

El primer ejemplo a tratar será un mosaico figurativo, concretamente el Mosaico de Atalanta y Meleagro $^{13}$, procedente de la villa romana de Cardeñajimeno (El Millar, Burgos).

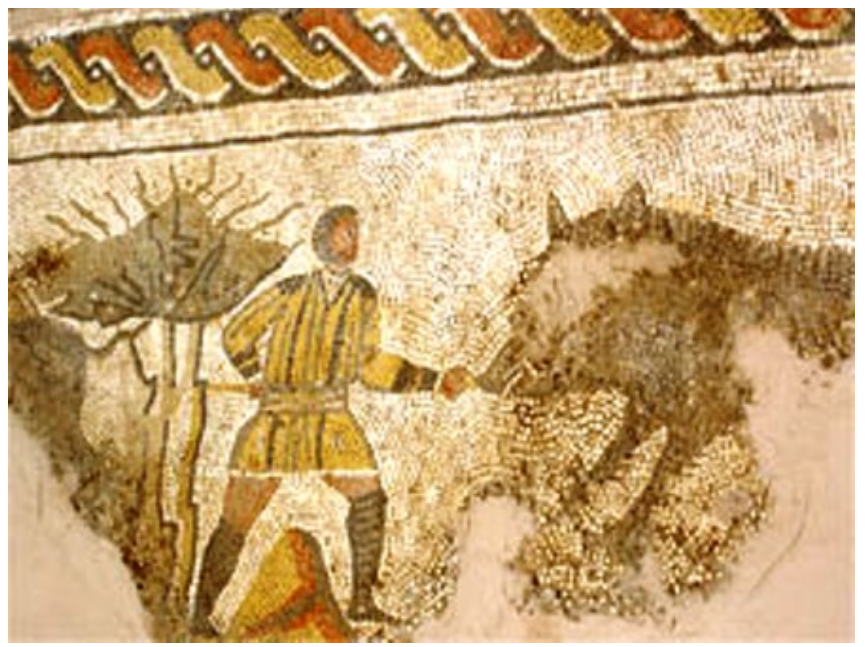

Fig. 1. Fragmento del Mosaico de Atalanta y Meleagro.

Meleagro ofreciendo a Atalanta el trofeo de jabalí de Calidon (en el oecus).

Fuente principal: https://burgospedial.files.wordpress.com/2012/10/0.jpg

La clasificación de Iconclass que se le ha aplicado a este mosaico es la siguiente:

\section{Clasificación Iconclass}

$25 \mathrm{~F}$ animals

\footnotetext{
11 Categorías: 0 Abstracto, Arte no figurativo / 1 Religión y la magia / 2 Naturaleza / 3 Ser Humano, el hombre en general / 4 Sociedad, Civilización, Cultura / 5 Ideas y conceptos abstractos / 6 Historia / 7 Biblia / 8 Literatura / 9 Mitología Clásica e Historia Antigua

12 Véase: http://www.iconclass.org/help/outline

13 Para ampliar información sobre el mosaico véase: https://musivariahd.com/mosaico-de-atalanta-y-meleagrocardenajimenolugarprocedencia-villa-cardenajimeno-el-millar-contexto-cultural-cultura-romanaconserv.html
} 


\section{Human Being, Man in General}

$41 \mathrm{C} 652$ vegetables

43C111241 stag-hunting

48A98783 floral interlace $\sim$ ornament

9 Classical Mythology and Ancient History

94N323 Meleager gives the head of the boar to Atalanta

La justificación de la clasificación utilizada en este caso sería la siguiente:

- Categoría 2: Naturaleza, dentro de ella la subcategoría 25F

- Categoría 3: Ser humano, hombre en general

- Categoría 4: Sociedad, civilización, cultura, y dentro de ella las subcategorías 41C, $43 \mathrm{C}$ y $48 \mathrm{~A}$

- Categoría 9: Mitología Clásica e Historia Antigua, dentro de ella la subcategoría 94N referente a las leyendas heroicas y la historia de Meleagro

Otro ejemplo, en este caso de mosaico geométrico, es el Mosaico ${ }^{\circ} 1$ con esvásticas y roseta de la villa romana de Requejo (Santa Cristina de la Polvorosa).

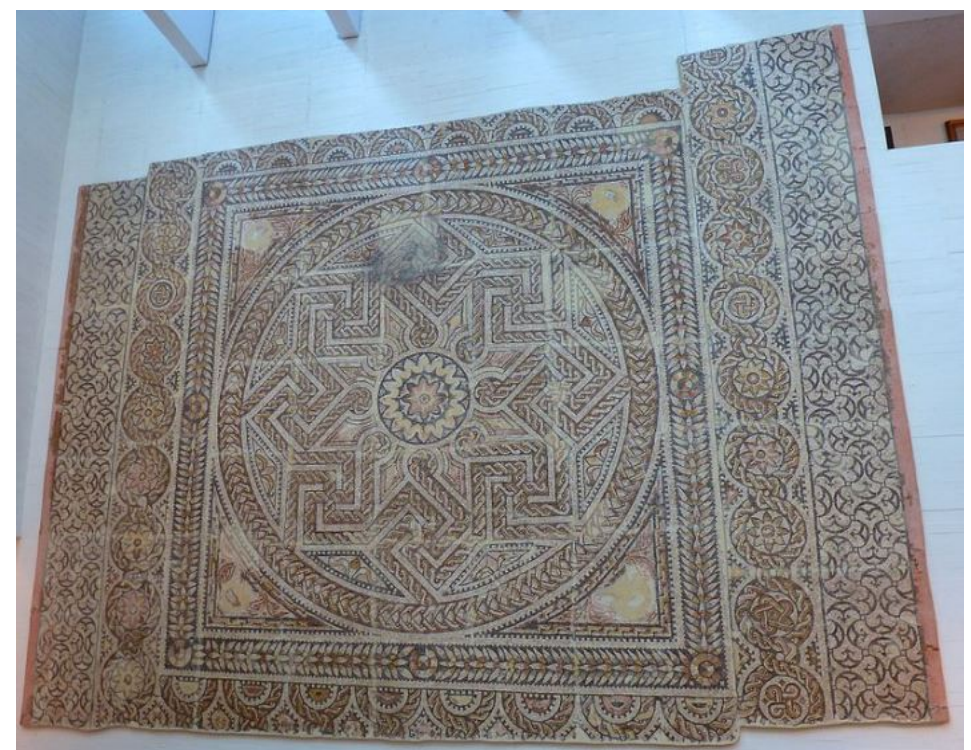

Fig. 2. Mosaico $\mathrm{N}^{\circ} 1$ con esvásticas y roseta central (Requejo, Zamora). Fotografía: Outisnn. Fuente:

https://es.wikipedia.org/wiki/Museo_de_Zamora\#mediaviewer/File:Mosaico_Requejo_grande.JPG

\section{Clasificación Iconclass}

11D123(SWASTIKA) specific forms of the cross: swastika 
48A981 ornament $\sim$ geometric motifs

48A98783 floral interlace $\sim$ ornament

48A98341 rosette $\sim$ ornament

$61 \mathrm{G}(. .$.$) names of artefacts and man-made objects (buildings excluded) (with NAME)$

La justificación de la clasificación utilizada en este caso sería la siguiente:

- Categoría 1: Religión y Magia, con la subcategoría 11D que este caso se refiere a una forma específica de cruz

- Categoría 4: Sociedad, civilización, cultura, y dentro de ella la subcategoría 48A que comparte en los tres casos la misma clasificación hasta 48A98 que se refiere a adornos $\sim$ arte

- Categoría 6: Historia, y dentro de ella la subcategoría $61 \mathrm{G}$ que se refiere a objetos con nombre concreto (excluye construcciones)

Como se puede observar, a pesar de tratarse de dos ejemplos de mosaico completamente diferentes, uno figurativo y otro geométrico, no por ello dejan de tener una clasificación concreta dentro de las categorías y subcategorías de Iconclass. Ambos obtienen notaciones que permiten hacerse una idea general, y según se avanza en la descripción particular (si se profundiza), del tema que albergan los mosaicos que describen.

Por este motivo, la clasificación ha resultado de utilidad en ambos tipos de mosaicos puesto que la misión ha sido la de describir la escena, independientemente de que fuera un tema mitológico por ejemplo, o por el contrario, una serie de objetos (como cráteras que abundan en los casos geométricos), motivos vegetales, grecas o entrelazados representados con frecuencia en los casos geométricos.

\subsection{Trabajo con metadatos}

En nuestra investigación hemos trabajado el objeto (mosaico), sus características y datos (descripción llevada a cabo con los metadatos y la clasificación Iconclass), el espacio en el que se encuentra el objeto (tipo de construcción que lo alberga) y sus dimensiones. Por ello, la selección de los metadatos ha resultado fundamental para la correcta clasificación de los mosaicos y su posterior recuperación.

A este respecto debemos comentar que se ha recurrido a Dublin Core Metadata Initiative ${ }^{14}$ (DCMI), cuyos metadatos se ajustan a estándares internacionales, de manera que permitió

\footnotetext{
14 Como se indica en la web: "Es una organización que apoya la innovación en el diseño de metadatos y las mejores prácticas en la ecología de los metadatos" (Traducción de la autora). http://dublincore.org/

Sus principios de funcionamiento son: Construcción de consenso abierto; Alcance y participación internacional; Neutralidad de propósitos y modelos comerciales; Neutralidad de la tecnología; Enfoque multidisciplinario. En cuanto a las especificaciones, se recomienda visitar los siguientes enlaces: https:/www.dublincore.org/specifications/dublin-core/ y http://dublincore.org/specifications/dublin-core/dcmi-terms/
} 
Musivaria HD: proyecto para el estudio, investigación y difusión de los mosaicos romanos tardoantiguos de la Península Ibérica desde las Humanidades Digitales

realizar un esquema de los datos en otros formatos, como por ejemplo en XML ${ }^{15}$ (eXtensible Markup Language). En consecuencia, los metadatos que se han utilizado para describir la información de las obras repertorizadas son los siguientes:

Tabla 1. Metadatos Dublin Core y correspondencia con la BD Musivaria HD

\begin{tabular}{|c|c|c|}
\hline Tipo & Metadato & Correspondencia en Musivaria HD \\
\hline Título & dc.title & Nombre del mosaico \\
\hline \multirow{3}{*}{ Ubicación } & \multirow{3}{*}{ dc.location } & $\begin{array}{c}\square \text { Lugar en el que se ubica el mosaico } \\
\text { (dentro del espacio) }\end{array}$ \\
\hline & & $\square$ Ubicación geográfica (lugar de procedencia) \\
\hline & & $\square$ Posición (pavimento o pared) \\
\hline Materia & dc.subject & Mosaico \\
\hline \multirow{16}{*}{ Descripción } & \multirow{16}{*}{ dc.description } & $\square$ Conservación (si o no) \\
\hline & & $\square$ Decoración (geométrica, \\
\hline & & figurativa o si contiene ambas) \\
\hline & & $\square$ Materia/soporte \\
\hline & & $\square$ Técnica \\
\hline & & $\square$ Color \\
\hline & & $\square$ Dimensión del mosaico \\
\hline & & y de la tesela \\
\hline & & $\square$ Estado de conservación \\
\hline & & $\square$ Descripción del mosaico \\
\hline & & $\square$ Historia \\
\hline & & $\square$ Iconografía \\
\hline & & $\square$ Epigrafía \\
\hline & & $\square$ Observaciones \\
\hline & & $\square$ Tema \\
\hline & & $\square$ Metadatos \\
\hline Periodo & dc.period & Periodo, cultura (tardoantiguo, romana) \\
\hline Periodo de tiempo & dc.periodoftime & Cronología \\
\hline Autor & dc.contributor.author & Beatriz Garrido-Ramos \\
\hline Fecha & dc.date.available & Fecha de creación \\
\hline Idioma & dc.language & Español \\
\hline Tipo & dc.type & Tipo de recurso (digital) \\
\hline Identificador & dc.identifier.uri & GeoNames, Iconclass, AAT/TAA \\
\hline
\end{tabular}

\footnotetext{
15 "Lenguaje de marcado para documentos. Permite definir una gramática por medio de un DTD (Document Type Definition) o un XML-Schema (esquema XML) utilizando etiquetas (metadatos) en todas sus combinaciones posibles" (Garrido-Ramos, 2019: 360). En la web W3C se expone lo siguiente: "Los esquemas XML expresan vocabularios compartidos y permiten que las máquinas ejecuten reglas creadas por personas. Proporcionan un medio para definir la estructura, el contenido y la semántica de los documentos XML. con más detalle". Véase: https://www.w3.org/XML/Schema

Las especificaciones ahora las mantiene el W3C XML Core Working Group.
} 


\subsection{Difusión del patrimonio cultural}

"Aunque el número de bienes catalogados se ha incrementado considerablemente, estos siguen siendo escasos si se tiene en cuenta el conjunto total del patrimonio cultural español" (Ladrón de Guevara, 2007: 1). En relación a esta cuestión cabe recordar que el caso de la musivaria no es una excepción.

El estudio de los mosaicos romanos, habitualmente, y salvo que se trate de equipos y/o excavaciones con grandes recursos, se suele llevar a cabo de manera aislada (individualizada), es decir, mediante el estudio de yacimientos concretos o incluso zonas en las que se ha efectuado algún descubrimiento.

La idea inicial era la de confeccionar una BD que albergara todos los mosaicos tardoantiguos de la Península Ibérica, labor de gran envergadura que por diversos motivos no pudo llevarse a la práctica. No obstante, se procedió a la selección de una muestra lo suficientemente representativa de mosaicos para el correspondiente estudio de toda la información por parte de la comunidad científica.

Durante el proceso, se valoró igualmente la necesidad de difundir el patrimonio musivo y una buena alternativa fue la creación de Musivaria HD. De esta forma, se presenta una parte de nuestro patrimonio material a toda persona interesada en el ámbito de los mosaicos romanos. La web, intuitiva y de sencillo manejo, ha dado a conocer desde su creación este arte romano que tanta información aporta de la época tardoantigua, ya sea a nivel social, cultural, geográfico o de otro tipo.

\subsection{Resultados}

El estudio de los mosaicos mediante la aplicación de su clasificación con Iconclass, además de una mejor recuperación de la información, ha permitido extraer la siguiente conclusión en relación a nuestra $\mathrm{BD}$. Se cumple la siguiente premisa: a mayor número de temas asignados a un mosaico mediante Iconclass menor número de casos existentes en los mosaicos de la BD. Esto demuestra que, a pesar de encontrar un repertorio variado, las escenas representadas tienden a reproducir menos temas en un mismo mosaico, procurando así los artistas no complicar demasiado la decoración musiva. Como indica el resultado, ese número de obras es infinitamente menor que las más habituales en las que se muestra una temática no compleja $\mathrm{y}$ de un grado de dificultad menor.

Igualmente, la recopilación, organización y procesado de la información, así como la visualización de los datos, ha permitido establecer nuevas categorías temáticas dentro de la iconografía representada en los mosaicos repertorizados. Así se ha contribuido a formular nuevas hipótesis y plantear otras líneas de investigación futuras para seguir avanzando en el estudio de la musivaria tardoantigua romana, no sólo de Hispania, sino también de otras partes del imperio. 


\section{Conclusiones}

La creación de la web Musivaria HD ha sentado un precedente en el estudio de la musivaria romana tardoantigua desde la perspectiva de las HD. Pionera en su campo, concentra una buena muestra de mosaicos, de España y Portugal, que nos invita a conocer mejor la época y la cultura romana a través de distinto tipo de documentación como reconstrucciones, vídeos, material descargable o vinculado entre sí (hipervínculo), etc.

Lo anterior ha sido posible gracias a un nuevo planteamiento y estudio de la iconografía representada en las escenas musivas. La interdisciplinariedad sumada a una nueva lectura con herramientas diversas, han permitido una mejor visualización que ha facilitado notablemente la comprensión por parte de los/as usuarios/as e investigadores/as.

A la primera fase de la investigación se añadió una segunda que se ha centrado en la descripción de la iconografía y en la que se ha trabajaron los metadatos y estándares. La aplicación de un sistema de clasificación como es el caso de Iconclass ha contribuido a un mejor conocimiento de los datos con los que partíamos al inicio de la investigación. Igualmente, la aplicación de estándares internacionales como el modelo CRM y Dublin Core han facilitado la interoperabilidad permitiendo también una mejor recuperación de la información.

Todo ello acompañado de un entorno amigable y comprensible para los usuarios, independientemente de sus conocimientos previos, permite una mejor experiencia entre los contenidos de la web, y por tanto, un mayor aprovechamiento de la misma contribuyendo así a la difusión de nuestro patrimonio.

\section{Referencias}

CIDOC-CRM (2014). Modelo de Referencia Conceptual (CRM). ISO 21127:2014.

Clasificación Iconclass. <http://www.iconclass.org/> [Consulta: 28 de enero de 2019]

DUBLIN CORE METADATA INITIATIVE. < http://dublincore.org/> [Consulta: 26 de enero de 2021]

GARRIDO-RAMOS, B. (2015). "Mosaicos en la Hispania Romana y Humanidades Digitales". En II Congreso Internacional: Innovación, globalización e impacto, Humanidades Digitales Hispánicas. Sociedad Internacional. Portal de Congresos y Conferencias de la UNED: Madrid. UNED. Disponible en <http://espacio.uned.es/congresosuned/index.php/hdh2015/hdh2015/paper/view/132> [Consulta: 1 de agosto de 2015]

GARRIDO-RAMOS, B. (2017a). "Propuesta de base de datos y catálogo de mosaicos tardoantiguos en la Hispania romana". UCOARTE Revista de Teoría e Historia del Arte, Vol. 6, pp.9-24. $<$ https://doi.org/10.21071/ucoarte.v6i.11001> [Consulta: 9 de octubre de 2018] 
GARRIDO-RAMOS, B. TaxMOS HD - Taxonomía para la investigación y difusión de la iconografia musivaria romana desde las Humanidades Digitales. < https://vocabularyserver.com/taxmos/> [Consulta: 25 de noviembre de 2019]

GARRIDO-RAMOS, B. (2017b). "Visualización de datos y clasificación Iconclass: un estudio de caso desde la perspectiva de las Humanidades Digitales". Caracteres. Estudios culturales y críticos de la esfera digital, $\quad$ Vol. 6010 (1), 6 . $<$ http://revistacaracteres.net/revista/vol6n1mayo2017/iconclass/> [Consulta: 5 de junio de 2018]

GARRIDO-RAMOS, B. (2019). Iconografia musivaria en la Península Ibérica en época romana: investigación y difusión desde el campo de las Humanidades Digitales. Tesis Doctoral. Madrid: UNED, Escuela Internacional de Doctorado (EIDUNED), Programa de Doctorado en Historia e Historia del Arte y Territorio, <http://e-spacio.uned.es/fez/view/tesisuned:ED-Pg-HHATBgarrido $>$ [Consulta: 20 de septiembre de 2020]

Diccionario Geográfico GeoNames. $<$ http://www.geonames.org/> [Consulta: 15 de enero de 2020]

LADRÓN DE GUEVARA, C. (2007). "La catalogación del patrimonio cultural: conceptos generales" en Seminario sobre la planificación de inventarios en centroamérica, San Salvador, 21-25 de mayo.

MANOVICH, L. (2008). "La visualización de datos como nueva abstracción y antisublime" en Estudios visuales: Ensayo, teoría y crítica de la cultura visual y el arte contemporáneo, 5, p. 126135.

Proyecto Musivaria $H D .<$ https://musivariahd.com/> [Consulta: 9 de enero de 2020]

RINCÓN ZAMORANO, M. (2015). "Introducción a la visualización de la información en humanidades digitales". Curso de Experto Profesional en Humanidades Digitales, UNED, 1, 2.

Tesauro de Arte y Arquitectura (TAA). <https://www.aatespanol.cl/> [Consulta: 26 de enero de 2021].

VV.AA. (2008). Manual de registro y documentación de Bienes culturales. Santiago de Chile: DIBAM, Getty. $\quad<$ https://www.cdbp.patrimoniocultural.gob.cl/652/articles-26006_archivo_01.pdf $>$ [Consulta: 29 de febrero de 2019]

XML. <https://www.w3.org/XML/Schema $>$ [Consulta: 26 de enero de 2021] 